\title{
CORRECTION
}

\section{Correction to: Fluid intake and urinary osmolality in pediatric patients with functional constipation}

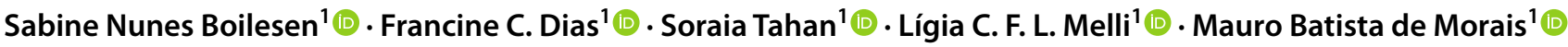

Published online: 13 October 2021

(c) Springer-Verlag GmbH Germany, part of Springer Nature 2021

\section{Correction to: European Journal of Nutrition}

https://doi.org/10.1007/s00394-021-02657-2

The original version of this article unfortunately contained a mistake in the row 'only boys' under the heading 'Dietary fiber (g/day)' and in the row 'only girls' under the heading 'Carbohydrate' (g/day).

The corrected Table 2 is given below.

The original article can be found online at https://doi.org/10.1007/ s00394-021-02657-2.

Mauro Batista de Morais

maurobmorais@gmail.com

Sabine Nunes Boilesen

sabine.boilesen@gmail.com

Francine C. Dias

francine.canovas@gmail.com

Soraia Tahan

s.tahan@uol.com.br

Lígia C. F. L. Melli

ligiamelli@gmail.com

Division of Pediatric Gastroenterology, Escola Paulista de Medicina, Universidade Federal de São Paulo, Rua Pedro de Toledo, 781-12 Andar, São Paulo, SP 04039-032, Brazil 
Table 2 Dietary data and water intake of children in the functional constipation group and control group

\begin{tabular}{|c|c|c|c|}
\hline & \multicolumn{2}{|l|}{ Group } & \multirow[b]{2}{*}{$p^{\mathrm{c}}$} \\
\hline & Constipation $^{\mathrm{a}}$ & Control $^{\mathrm{b}}$ & \\
\hline \multicolumn{4}{|c|}{ Energy and macronutrient intake } \\
\hline \multicolumn{4}{|c|}{ Energy (Kcal/day) } \\
\hline Girls and boys & $1493.0(1096.3 ; 1838.8)$ & $1853.0(1412.5 ; 2492.0)$ & 0.003 \\
\hline Only girls & $1365.0(906.0 ; 1527.0)$ & $1610.0(1303.0 ; 2434.8)$ & 0.033 \\
\hline Only boys & $1540.0(1102.5 ; 1928.0)$ & $2092.0(1586.0 ; 2531.0)$ & 0.005 \\
\hline \multicolumn{4}{|c|}{ Carbohydrate (g/day) } \\
\hline Girls and boys & $208.6(153.8 ; 264.2)$ & $261.1(186.9 ; 370.3)$ & 0.008 \\
\hline Only girls & $179.6(143.1 ; 250.1)$ & $226.8(173.7 ; 355.4)$ & 0.078 \\
\hline Only boys & $210.4(168.9 ; 286.1)$ & $313.2(233.8 ; 382.6)$ & 0.011 \\
\hline \multicolumn{4}{|l|}{ Protein (g/day) } \\
\hline Girls and boys & $59.3(38.2 ; 87.3)$ & $82.5(54.0 ; 103.8)$ & 0.006 \\
\hline Only girls & $53.1(34.1 ; 79.2)$ & $76.4(49.2 ; 102.7)$ & 0.061 \\
\hline Only boys & $60.0(38.6 ; 89.1)$ & $83.7(61.0 ; 108.7)$ & 0.013 \\
\hline \multicolumn{4}{|l|}{ Fat (g/day) } \\
\hline Girls and boys & $42.2(30.8 ; 57.2)$ & $48.7(36.5 ; 66.2)$ & 0.142 \\
\hline Only girls & $35.9(22.5 ; 55.2)$ & $44.1(28.8 ; 64.9)$ & 0.247 \\
\hline Only boys & $43.0(33.3 ; 66.2)$ & $55.0(40.9 ; 67.4)$ & 0.110 \\
\hline \multicolumn{4}{|c|}{ Dietary fiber (g/day) } \\
\hline Girls and boys & $16.8(11.9 ; 21.6)$ & $23.8(15.5 ; 33.1)$ & 0.014 \\
\hline Only girls & $17.1(14.7 ; 20.8)$ & $23.3(15.5 ; 37.7)$ & 0.078 \\
\hline Only boys & $16.7(10.2 ; 26.2)$ & $23.9(14.3 ; 38.6)$ & 0.066 \\
\hline \multicolumn{4}{|l|}{ Water intake } \\
\hline \multicolumn{4}{|c|}{ Tap water (plain water) (mL/day) } \\
\hline Girls and boys & $500.0(250.0 ; 1000.0)$ & $1,000.0(520.0 ; 1,350.0)$ & 0.016 \\
\hline Only girls & $750.0(200.0 ; 1100.0)$ & $800.0(550.0 ; 1500.0)$ & 0.128 \\
\hline Only boys & $500.0(250.0 ; 1000.0)$ & $1000.0(510.0 ; 1000.0)$ & 0.021 \\
\hline \multicolumn{4}{|c|}{ Water from beverages and juices (mL/day) } \\
\hline Girls and boys & $666.8(321.0 ; 917.4)$ & $812.3(593.5 ; 1018.2)$ & 0.021 \\
\hline Only girls & $609.4(276.6 ; 883.5)$ & $814.2(514.2 ; 1062.0)$ & 0.011 \\
\hline Only boys & $727.7(573.9 ; 1055.9)$ & $789(628.7 ; 1006.7)$ & 0.821 \\
\hline \multicolumn{4}{|c|}{ Water contained in foods (mL/day) } \\
\hline Girls and boys & $293.3(182.1 ; 415.3)$ & $370.1(245.1 ; 539.5)$ & 0.025 \\
\hline Only girls & $296.4(186.2 ; 419.1)$ & $380.6(199.3 ; 535.0)$ & 0.132 \\
\hline Only boys & $290.2(154.3 ; 410.4)$ & $368.0(264.1 ; 544.7)$ & 0.172 \\
\hline \multicolumn{4}{|c|}{ Total water (mL/day) } \\
\hline Girls and boys & $1649.8(1244.1 ; 2010.0)$ & $2177.8(1598.9 ; 2581.4)$ & $<0.001$ \\
\hline Only girls & $1651.0(1283.3 ; 2109.1)$ & $2354.5(1617.6 ; 2724.6)$ & 0.008 \\
\hline Only boys & $1648.5(1137.5 ; 2015.9)$ & $2149.5(1459.7 ; 2469.8)$ & 0.045 \\
\hline \multicolumn{4}{|c|}{ Total water/energy (mL/Kcal) } \\
\hline Girls and boys & $1.0(0.8 ; 1.7)$ & $1.1(0.9 ; 1.5)$ & 0.562 \\
\hline Only girls & $1.0(0.8 ; 1.3)$ & $1.1(0.9 ; 1.3)$ & 0.682 \\
\hline Only boys & $1.2(0.7 ; 1.8)$ & $1.3(0.9 ; 1.5)$ & 0.993 \\
\hline \multicolumn{4}{|c|}{ Total water/dietary fiber (mL/g) } \\
\hline Girls and boys & $97.4(59.8 ; 153.6)$ & $94.4(66.2 ; 142.9)$ & 0.969 \\
\hline Only girls & $91.3(61.0 ; 184.1)$ & $105.3(68.0 ; 150.7)$ & 0.905 \\
\hline Only boys & $110.6(54.7 ; 139.5)$ & $88.4(66.1 ; 136.4)$ & 0.878 \\
\hline
\end{tabular}

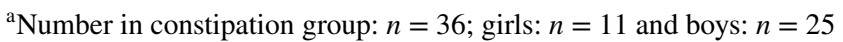

bumber in control group: $n=93$; girls: $n=52$; boys: $n=41$

${ }^{\mathrm{c}}$ Mann-Whitney test; median and percentiles 25 and 75 